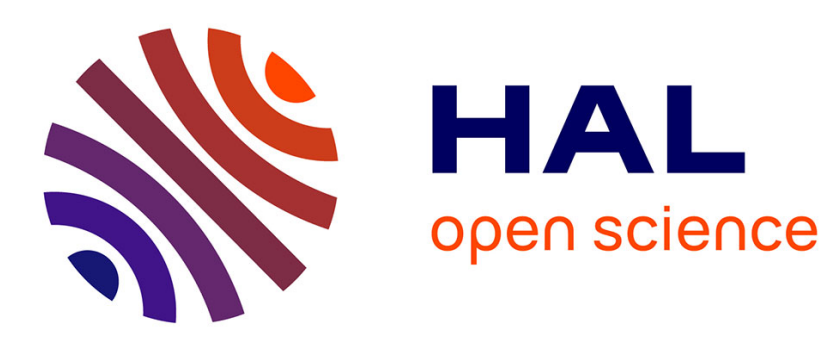

\title{
Effects of anisotropy on the equilibrium shape of nanoscale pores at grain boundaries \\ P. Wynblatt, D. Chatain
}

\section{To cite this version:}

P. Wynblatt, D. Chatain. Effects of anisotropy on the equilibrium shape of nanoscale pores at grain boundaries. Acta Materialia, 2013, 61 (12), pp.4572-4580. 10.1016/j.actamat.2013.04.025 . hal00844986

\section{HAL Id: hal-00844986 \\ https://hal.science/hal-00844986}

Submitted on 6 May 2018

HAL is a multi-disciplinary open access archive for the deposit and dissemination of scientific research documents, whether they are published or not. The documents may come from teaching and research institutions in France or abroad, or from public or private research centers.
L'archive ouverte pluridisciplinaire HAL, est destinée au dépôt et à la diffusion de documents scientifiques de niveau recherche, publiés ou non, émanant des établissements d'enseignement et de recherche français ou étrangers, des laboratoires publics ou privés. 


\title{
Effects of anisotropy on the equilibrium shape of nanoscale pores at grain boundaries
}

\author{
P. Wynblatt ${ }^{\mathrm{a}}$ and D. Chatain ${ }^{\mathrm{b}}$ \\ ${ }^{a}$ Department of Materials Science and Engineering, Carnegie Mellon University, Pittsburgh, \\ PA 15213, USA. \\ email:pw01@andrew.cmu.edu \\ ${ }^{\mathrm{b}}$ Aix-Marseille University, CNRS, CINaM, F-13288 Marseille, France. \\ email: chatain@cinam.univ-mrs.fr
}

\begin{abstract}
Molecular dynamics simulations have been performed to study the interaction between a faceted pore and an anisotropic grain boundary (GB). Nickel was chosen as a convenient model system. In order to establish the equilibrium crystal shape (ECS) of the pore, studies were also conducted on isolated pores. Isolated pores were found to be subject to the nucleation inhibition of equilibration that has been predicted by Rohrer et al. [J Am Ceram Soc 2000;83:214, 2001;84: 2099]. This work shows that configurations close to the ECS can be obtained if supersaturation within a pore is artificially increased by adding mobile adatoms to the internal surfaces of the pores. In the case of pores located at GBs, the nucleation energy barriers to facet displacement are not present for facets in contact with the GB at the triple line, but may still persist for facets that have no contact with the GB. This problem can be overcome by approaching the equilibrium shape from different initial configurations. The configuration of the GB in the vicinity of the pore has been found to be essentially planar, indicating that GB puckering in the vicinity of anisotropic pores is not generally necessary. The present calculations show that incompatibilities between misoriented pore facets that meet at the triple line with the GB are easily accommodated by local atomic rearrangements at the disordered region of intersection with the GB.
\end{abstract}

Keywords: atomistic simulation; nanopores; grain boundaries; morphology; triple line 


\section{Introduction}

Sintered materials commonly contain pores. The pores are transient microstructural features, as they must ultimately disappear by diffusion of vacancies away from their surfaces towards sinks such as dislocations, grain boundaries and/or external surfaces, so as to reduce the excess free energy associated with their internal surfaces. Nevertheless, the kinetics of these processes can be sufficiently slow for pores to persist, and to play a significant role in the evolution of the microstructure of the sintered material. For example, the presence of pores at grain boundaries (GBs) can inhibit grain growth, because movement of a GB away from a pore requires an increase in interfacial energy associated with restoration of the GB area that was eliminated when the GB intercepted the pore. This phenomenon is known as Zener pinning.

Crystalline materials generally possess anisotropic surface energy. This anisotropy is reflected in the equilibrium crystal shape (ECS) of the material, which is related to the surface energy anisotropy by the Wulff construction [1-3]. A pore in such a material may be viewed as a "negative crystal" and will develop a shape that is geometrically similar to the equilibrium crystal shape. Pores intercepted by GBs will display part of the complete ECS in each of the two crystals separated by the GB, and these partial ECSs will be misoriented with respect to each other by the same misorientation as that which characterizes the grain boundary [4-6].

In two-phase crystalline materials, there have been some previous investigations of the manner in which a GB intercepts a solid second phase particle (precipitate) that displays anisotropy of the particle/matrix interfacial energy [7-10]. In order to accommodate the particle equilibrium shape, the triple line joining the particle and the GB may not remain planar, with a consequent "puckering" of the GB in the vicinity of the particle. However, the extent to which a GB may pucker will depend on the GB energy as well as its anisotropy (i.e. stiffness).

The purpose of the present paper is to investigate, by molecular dynamics simulations, the manner in which the shape of a pore in a material with anisotropic surface energy responds to its interaction with a GB, and correspondingly how the GB shape responds to its interaction with the pore.

\section{Computational approach}

Molecular dynamics (MD) simulations were conducted primarily by means of the LAMMPS code $[11,12]$, and occasionally by means of the PARADYN code $[13,14]$, which 
is a precursor of LAMMPS developed explicitly for use with embedded atom method (EAM) potentials. Both codes were employed in conjunction with the Foiles-Hoyt EAM potential for nickel [15]. Computations were performed for a constant number of atoms, constant pressure and constant temperature, (i.e., a so-called NPT ensemble). The desired pressure was set to zero.

Most of the work was performed on pores residing at a $\Sigma 13$ twist GB. The GBs were created by relative rotation of the two adjoining crystals about the [001] direction. The $\Sigma 13$ GB was selected based on recent calculations by Olmsted et al. [16] of the energies of 388 twist GBs, which made use of the same interatomic potential. That work showed that the $\Sigma 13$ twist GB had a somewhat higher than average energy among twist GBs terminated on $\{100\}$ planes. Therefore it is likely that the $\Sigma 13$ GBs do not lie at a deep cusp in GB-energy versus orientation space. This feature implies that such GBs should not display large torques, and are therefore more likely to change their inclinations in the vicinity of the pore to accommodate any tendency of the GB to pucker. This issue will be the subject of further discussion below.

A schematic of the computation cells used in most of the simulations is shown in Fig. 1. The initial configurations of the $\Sigma 13$ GBs used in the present calculations were formed by adjoining two perfect half-crystals at appropriate misorientations, and then replacing any atom pairs closer than 0.5 interatomic distances by a single atom at the mean position of the pair. Two types of pores were studied. Most of the computations were conducted on initially spherical pores (Fig. 1). In this case, each of the two perfect half-crystals contained a hemispherical cavity $15 \mathrm{a}_{0}$ (where $\mathrm{a}_{0}$ is the lattice constant), or $5.28 \mathrm{~nm}$, in diameter. In other cases, the initial form of the pore was taken to be lens-shaped. These pores also had an initial diameter of $15 \mathrm{a}_{0}$ at their intersection with the GB, but only a height of $7.5 \mathrm{a}_{0}$ in the direction perpendicular to the GB, compared with a height of $15 \mathrm{a}_{0}$ for the initially spherical pores. In addition, calculations were conducted for two different GB areas. In most computations the area of the GB was $\sim 115 \mathrm{~nm}^{2}$ for a total number of atoms in the computation cell of $\sim 10^{5}$. A few computations were performed with a GB area of $\sim 225 \mathrm{~nm}^{2}$ and a total number of atoms of $\sim 4 \times 10^{5}$. The reasons for these choices will be discussed below.

Simulations of the pore-GB system were performed with 3-D periodic boundary conditions, so that additional GBs were present at the edges parallel to the GB. In order to prevent the edge GBs from distorting the pore and central GB, the atoms on the edges were constrained to have zero velocity, but their positions were scaled with changes in the 
dimensions of the computation cell due to temperature changes and the requirement of zero pressure. Simulations were conducted between 1200 and $1350 \mathrm{~K}$.

In addition, simulations were performed to investigate the shape of an isolated pore (of the same initial size as that of the initially spherical pore at the GB) so as to determine the ECS of the pore in the absence of a GB.

\section{Configurations and Interfacial Energies}

It is convenient to introduce some background that will be useful in the subsequent analysis of the configurations obtained in the simulations, and in particular to provide some relationships that link those configurations to the energies of the interfaces involved. Consider the configuration of an isolated faceted pore. According to the Wulff construction [1-3], the ECS of a pore is dictated by the relative energies of the surfaces that surround it, i.e.:

$$
\frac{\gamma_{h k l}}{d_{h k l}}=K
$$

where $\gamma_{h k l}$ and $d_{h k l}$ are the surface energy and distance from the center of the ECS (or Wulff point) to the surface of orientation $h k l$, respectively, and $K$ is a constant. Thus, examination of the shape of an equilibrated pore will yield the relative surface energies of all surfaces that surround the pore. While it could be useful to have values of these surface energies a priori, e.g. to assess the degree of equilibration of the pore, this is not essential. In particular, the evaluation of individual surface energies at the temperatures of interest is not a trivial task, and typically requires computation-intensive thermodynamic integration. Thus, in the present study we have made use of surface energies calculated at $0 \mathrm{~K}$ by lattice statics calculations, in order to estimate surface energies ratios at temperature. This is clearly an approximation, but one that is quite reliable, in view of the weak temperature dependence of surface energies.

We now turn to the case of the equilibration of a pore with a GB. To begin with, we consider the expected equilibrium behavior of a pore at a GB for the case in which the pore displays isotropic surface energy. Under those conditions, the balance of interfacial energies yields the well-known relation:

$$
\gamma_{G B}=2 \gamma_{S} \cos \theta
$$

where $\gamma_{G B}$ and $\gamma_{s}$ are the grain boundary and isotropic surface energy, respectively, and $\theta$ is half the dihedral angle, as shown schematically in Fig. 2a. In addition to illustrating the expected lenticular shape for the pore, the figure includes the ECSs of the pore edges in each 
of the adjoining crystals. Since the ECSs for this isotropic case are identical with the Wulff plots, the radii of the ECS for either the lower or upper edges of the pore also represent the surface energy of the pore (to within an arbitrary scale factor). Thus, the distance from the Wulff point to the GB plane, $a$, may be written as:

$$
2 a=2 \gamma_{S} \cos \theta
$$

Comparing Eqs. 2 and 3, it is clear that the separation of the Wulff points of the upper and lower edges of the pore is proportional to the GB energy (to within the same arbitrary scaling factor as the surface energy). This result, illustrated here for the case of a pore at a GB, applies equally well to the case of a precipitate at a GB. Also, in this isotropic case, there is no driving force for puckering of the GB.

The above approach can be extended to the case of GB pores with anisotropic surface energies [7, 8, 17]. If the anisotropic case displayed in Fig. $2 b$ is considered, the corresponding result is that the distance separating the Wulff points of ECSs of the pore edges is also proportional to the energy of the GB. Making use of Eq. 1, and recognizing that $\left(a+a^{\prime}\right)=2 a$ :

$$
\frac{b}{c}=\frac{c-2 a}{c}=\frac{2 \gamma_{100}-\gamma_{G B}}{2 \gamma_{100}}=1-\frac{\gamma_{G B}}{2 \gamma_{100}} \equiv 1-\cos \theta_{e f f}
$$

where the pore surfaces that lie parallel and perpendicular to the plane of the GB in Fig. 2b have been taken to be the $\{100\}$ pore surfaces, in accordance with our simulations of pores on twist GBs (see Fig. 4 below), and $\theta_{\text {eff }}$ is defined as an "effective contact angle" for the anisotropic case, by analogy with Eq. 2 for the isotropic case.

The main purpose of the above description of the configuration of a pore at a GB is to indicate that the shape of the pore depends on both the interfacial energies of the surfaces surrounding the pore as well as the GB energy. Thus again, it could be useful to have an independent calculation of the GB energy at the temperature of the simulations. However, it has recently been shown by lattice statics calculations, that even at $0 \mathrm{~K}$, identification of the GB configuration that corresponds to minimum energy might require investigation of several thousand GB configurations [16]. Thus, comparable studies at finite temperatures would present a formidable task, and have not been attempted. When required in the present work, we have therefore used $0 \mathrm{~K}$ value of the $\Sigma 13$ twist GB energy computed by Olmsted et al. [16]. Again, it should be stressed that this approximation is quite reliable since, as in the case of surfaces, the temperature dependence of GB energy is rather weak. 


\section{Results and Discussion}

\section{$\underline{4.1 \text { Configuration of Isolated Pores }}$}

After performing MD simulations of an isolated pore for a given time at a given temperature, the structure was relaxed at $0 \mathrm{~K}$ by lattice statics energy minimization to eliminate thermal noise. The following scheme was applied to the relaxed structure to display the pore configuration. Coordination numbers of the atoms in the computational cell were calculated by using a cut-off distance half way between first and second neighbors. The only atoms displayed are those that possess a coordination lower than the bulk value of 12 . Some examples are shown in Fig. 3. These show the atomic structure of the pore exterior as viewed along a $<100>$ direction.

Figure 3(a) displays the structure of the initial "spherical" pore configuration prior to MD equilibration. This configuration was produced by cutting a spherical cavity $\left(15 \mathrm{a}_{0}\right.$ in diameter) out of the perfect lattice. However, because of the atomically discrete nature of the pore, it shows several facets: one $\{100\}$ facet (yellow), four $\{111\}$ facets (brown), four $\{311\}$ facets (blue-green) lying between the $\{100\}$ and $\{111\}$ facets, and four $\{110\}$ facets (multicolored) lying between adjacent $\{111\}$ facets. Unlike other facets, the $\{110\}$ facets display a few adatoms as a result of the spherical shape of the pore.

Figure 3(b) shows a structure obtained after MD equilibration for $20 \mathrm{nsec}$ at $1350 \mathrm{~K}$, followed by relaxation at $0 \mathrm{~K}$. Several features are apparent: the $\{100\}$ and $\{111\}$ facets have increased in size, the $\{311\}$ facets have almost disappeared, and narrow disordered $\{110\}$ facets are present. In addition, the $\{111\}$ facets display inverse pillboxes, which appear as monoatomic height protrusions when viewed from the exterior of the pore. Some of these protrusions are due to "magic size" effects [18], which arise from the arbitrary choice of cutting out a cavity $15 \mathrm{a}_{0}$ in diameter to produce the initial configuration of the computation cell. Thus, the number of atoms removed in the creation of the pore will generally not match the size of a cavity that will produce a pore with a perfectly shaped ECS.

Given the relation between the surface energies of a faceted pore described in Eq. 1, there is no compelling reason for the ECS to adopt a perfectly symmetric shape, as the relative distances of surfaces of different orientations will be in the ratio of their surface energies, which need not coincide exactly with a perfectly symmetric atomically discrete cavity. Nevertheless, after $20 \mathrm{nsec}$ equilibration at $1350 \mathrm{~K}$, Fig. $2 \mathrm{~b}$ still displays an uncentered $\{100\}$-type facet, and $\{111\}$ facets that are significantly different in size. This lack of equilibration of isolated faceted pores is related to the existence of nucleation energy barriers that inhibit the displacement of facets, as has been described by Rohrer et al. [19, 20]. 
They have shown that for facet displacement in the direction of its normal, it is necessary to form a pillbox-shaped nucleus at the facet surface. In the case of a faceted crystal that has achieved its equilibrium shape, the nucleation energy barrier for either growth or shrinkage of the facet is identical. However, for a non-equilibrium faceted shape, the magnitude of the nucleation barrier depends on whether a given facet needs to grow or to shrink in order to approach equilibrium; but in either case, the supersaturation needed to drive the nucleation process becomes too small well before the faceted crystal has reached its equilibrium shape. As a result, Rohrer et al. have concluded that faceted crystals are unlikely to reach their equilibrium shapes in the absence of certain defects, such as for example, dislocations with a screw component that thread the facet surface, or GBs that are in contact with the facet and are able to provide a disordered region that can act as pre-existing steps.

In order to aid in the equilibration process in the present study, we have artificially increased the supersaturation in the pores by adding some adatoms to the internal pore surface. Figure 3c shows a structure obtained by MD equilibration for $12 \mathrm{nsec}$ at $1300 \mathrm{~K}$, after adding 400 randomly distributed adtoms to the surface of the pore of Fig. $3 \mathrm{~b}$. The structure displayed in Fig. $3 \mathrm{c}$ has also been relaxed at $0 \mathrm{~K}$ to reduce thermal noise. Although this structure still contains some deviations from symmetry, it is clearly more symmetric than the pore of Fig. 3b. Some of the remaining structural irregularities are due to the high equilibration temperature, which is bound to produce some thermal defects. Nevertheless, Fig. $3 \mathrm{c}$ gives a reasonable idea of the pore ECS at $1300 \mathrm{~K}$. It consists of large $\{100\}$ and $\{111\}$ facets, small $\{110\}$ facets that still contain some thermal defects. Shapes similar to Fig. 3c were also obtained from MD simulations for $12 \mathrm{nsec}$ at $1350 \mathrm{~K}$.

Figure 4 compares the rate of pore equilibration by examining the ratio $\gamma_{100} / \gamma_{111}$ as a function of time for pores of the type shown in Fig. $3 b$ (no added supersaturation) and those of Fig. 3c (with 400 atoms added to the pore surface to increase supersaturation). Fig. 4a shows that equilibration is slow for pores without enhanced supersaturation, and that the ratio of $\gamma_{100} / \gamma_{111}$ has still not reached an asymptotic value even after $20 \mathrm{nsec}$ at $1350 \mathrm{~K}$. This lack of equilibration is consistent with the asymmetric configurations of the type illustrated in Fig. 3b. On the other hand, by adding a few high-mobility adatoms, one finds that the surface energy ratio approaches an asymptotic limit of $\gamma_{100} / \gamma_{111}=1.07$ after $12 \mathrm{nsec}$ at $1350 \mathrm{~K}$, as shown in Fig. $4 b$. 
For the purposes of comparison with the results of Fig. 4, the energies of the $\{100\}$ and $\{111\}$ surfaces were calculated by lattice statics simulations at $0 \mathrm{~K}$, and yielded a ratio of $\gamma_{100} / \gamma_{111}=1.055$. This is in reasonable agreement with the results of Fig. $4 \mathrm{~b}$.

\subsection{Configuration of Pores on Grain Boundaries}

The primary interest of this study is morphological; it is to determine how the ECS of anisotropic pores is modified by the intersection with GBs, and how the shape of GBs may be affected by their interactions with pores. The detailed energetics of such systems are not of direct interest. The GBs used in the simulations were constructed without seeking to ensure that the initial GB structure had the minimum energy configuration.

The arbitrary manner in which the initial GB structure was selected in the present study is unlikely to produce, fortuitously, the minimum GB energy. However, as stated in the introduction, a pore is intrinsically unstable. Thus, there will be tendency for the pore to absorb any excess atoms present in the GB in the process of approaching equilibrium during MD simulation. This is useful, as the GB can act as a natural source of atoms that will aid in reaching local equilibrium between the GB and the pore, in a manner analogous to the procedure used in the study of isolated pores, in which free adatoms were added to the pore surface to accelerate equilibration. A typical result of the configurations obtained from the simulations with an initially spherical pore is shown in Fig. 5.

The figure shows the two-parts of a pore, separated by a $\Sigma 13$ GB (only part of the GB used in the simulation is displayed in the figure). From a comparison with Fig. 3c, it is should be clear that the upper and lower parts of the pore amount to somewhat less than half of the ECS of an isolated pore. The two halves of the pore are rotated by $\sim 22^{\circ}$ with respect to each other about an axis perpendicular to the GB, in accordance to the misorientation of the $\Sigma 13$ GB. In the viewing direction of the figure, the upper part of the pore is rotated $11^{\circ}$ to the right and its lower part is rotated $11^{\circ}$ to the left. The top and bottom faces of the pore (seen edgeon) lie on $\{100\}$ surfaces. The figure also shows a vacancy in the bulk of the upper crystal (small isolated cube-shaped feature in upper right).

In order to analyze configurations such as Fig. 5, we have computed the separation distance between the two $\{100\}$ facets at the top and bottom of the pore (i.e. dimension $b$ in the schematic of Fig. 2) and the separation distance of the two $\{100\}$ facets on the right and left edges of the upper and lower parts of the pore (dimension $c$ in Fig. 2). The ratio of $b / c$ corresponds to $\left(1-\cos \theta_{e f f}\right)$, as indicated in Eq. 4. However, the schematic of Fig. 2 was 
drawn for a "macroscopic" case, in which the GB is assumed to be a plane of zero thickness. In the present "atomistic" simulations, where the pore dimensions are only a few nm, it would be improper to ignore the fact that the GB has a finite free volume, which expands the computation cell in the direction perpendicular to the GB plane. We have estimated this expansion in the dimension $b$ by comparing relaxed configurations with and without a GB and find that it amounts to $\sim 0.4 \mathrm{~nm}$. Thus, the dimension $b$ has been corrected for this expansion in the analysis given below.

In order to compute the positions of the $\{100\}$ faces that define the pore dimensions $b$ and $c$, the average positions of atoms having coordination numbers of 8 in the vicinity of the facets of interest were computed. The results for the value of $b / c$, corrected for GB free volume, are displayed in Fig. 6. The figure shows that after $1 \mathrm{nsec}$ at $1200 \mathrm{~K}$ the value of $\mathrm{b} / \mathrm{c}$ is greater than 1, which according to Eq. 4, cannot correspond to equilibrium and is physically unrealistic. The reason for this result is that the $\{100\}$ facets that are in contact with the GB are not subject to the nucleation barriers, which can impede facet motion in the absence of pre-existing defects $[19,20]$. These facets are in contact with the disordered GB and can undergo displacement much more rapidly than the top and bottom $\{100\}$ facets, which must still undergo nucleation events in order to move. Thus, the dimension $c$ changes (shrinks) more rapidly than the dimension $b$, leading to pore shapes that are far from equilibrium. However, by increasing the simulation temperature and providing additional time, the dimension $b$ of the pores eventually also undergoes shrinkage, thus reducing the ratio $b / c$ to acceptable values below unity.

It is possible to estimate the equilibrium value of $b / c$ (i.e., $1-\cos \theta_{\text {eff }}$ ) from the values of the $\{100\}$ surface energy and the GB energy computed at $0 \mathrm{~K}: \gamma_{100}=2.050 \mathrm{~J} / \mathrm{m}^{2}$, and $\gamma_{G B}$ $=0.884 \mathrm{~J} / \mathrm{m}^{2}$ [16]. These values yield $b / c=0.78$, which is significantly smaller than the lowest value of $\sim 0.9$ obtained in Fig. 6 after apparent equilibration at $1350 \mathrm{~K}$. In view of the expected nucleation inhibition of the equilibration kinetics [19, 20], it was considered useful to approach equilibrium from an initially lower value of $b / c$. Thus, simulations were conducted starting from an initial configuration consisting of a lenticular pore having a diameter of $15 \mathrm{a}_{0}$ at the GB plane, and a height in the perpendicular direction of $7.5 \mathrm{a}_{0}$ (i.e. an initial value of $\theta_{\text {eff }}=60^{\circ}$, rather than the value of $\theta_{\text {eff }}=90^{\circ}$ in the case of an initially spherical pore). The results of these simulations are shown in Fig. 7. The figure shows that the ratio $\mathrm{b} / \mathrm{c}$ reaches a value of $\sim 0.75$ after $16 \mathrm{nsec}$ of MD at $1300 \mathrm{~K}$, and increases to about 0.8 after an additional $4 \mathrm{nsec}$ at $1350 \mathrm{~K}$. Unfortunately, it was not possible to extend the computations 
at $1350 \mathrm{~K}$, because for times longer than $\sim 4 \mathrm{nsec}$, the lenticular pore vanishes, i.e. it is filled up by atoms originating from the GB. Nevertheless, the value of $b / c$ achieved in this case is much closer to the value of 0.78 expected from the interfacial energies computed at $0 \mathrm{~K}$. Thus, we take the configuration obtained from the initially lenticular pore shape to be close to the ECS of the pore at the GB.

\section{$\underline{4.3 \text { Configuration of GBs with Pores }}$}

When a pore (or a precipitate) is present at a GB, the lowest energy configuration may involve "puckering" of the GB [7-10]. Any deviation of the GB from a planar configuration will increase the GB area, so that the GB energy component of the system will increase. This component could also increase, through the Gibbs-Thomson effect, if puckering involves GB curvature. Any increase in the energy associated with the GB would need to be offset by a larger decrease in the interfacial energy of the pore and/or the energy of the triple line at which the GB connects to the pore.

Most of the computations of the pore-GB system described above were performed with a GB area of $\sim 115 \mathrm{~nm}^{2}$, i.e. with linear dimensions of approximately twice the pore diameter. However, the smaller the GB area in relation to the pore size, the larger the local curvatures that might be necessary for a GB to pucker, i.e. small GB dimensions could conceivably constrain the GB into adopting high curvatures in the vicinity of the pore. Thus, the computations aimed at investigating the possibility of GB puckering were performed with a doubled GB area of about $225 \mathrm{~nm}^{2}$, in order to avoid constraints arising from GB curvature effects.

The GB energies predicted by the EAM potential used here are naturally anisotropic [16]. In this case, the appropriate form of the Gibbs-Thomson equation for the relationship between chemical potential change and GB curvature is [21]:

$$
\Delta \mu=\Omega\left(\gamma+\gamma^{\prime \prime}\right) \kappa
$$

where $\mu$ is the chemical potential, $\Omega$ is the atomic volume, $\gamma$ " is the second derivative of the surface energy with respect to orientation, $\kappa$ is the curvature, and the quantity $\left(\gamma+\gamma^{\prime \prime}\right)$ is generally referred to as the GB stiffness. Although the stiffness is a tensor property [22], for the present purposes it is sufficient to consider a one-dimensional case.

The following procedure was used to define the configuration of a GB surrounding a pore. The GB region was divided into square area elements $1 \mathrm{~nm}^{2}$ in size. Only atoms with a coordination number of 11 were considered within these area elements, as these constitute the 
majority of atoms belonging to the GB. The z-dimensions of all atoms falling within these area elements were averaged, and a contour plot of the average heights in the direction perpendicular to the GB plane was obtained. An example of such a plot is shown in Fig. 8. Examination of the figure shows that the GB is fairly flat in the vicinity of the pore, and shows no obvious systematic puckering. In contrast, there are several regions at large distances from the pore that show substantial local curvature, e.g. in the upper left hand side of the figure where the radius of curvature is about $10 \mathrm{~nm}$. This relatively high curvature occurs under the influence of the weak forces due to thermal fluctuations, and therefore demonstrates that the GB stiffness is too low to inhibit GB puckering. Thus, we conclude that for the system studied here, the configuration of the pore-GB system can reach equilibrium without any detectable GB puckering.

It is difficult to view the structure of the triple line surrounding the pore at its intersection with the GB from figures such as Fig. 5, as the atoms of the GB are superimposed on exterior of the pore and conceal the triple line. However, it is possible to view the triple line from the pore interior by slicing the pore. Such a structure is shown in Fig. 9.

The figure shows that although the two $\{100\}$ (yellow) facets that adjoin the triple line are rotated about the (vertical) z-axis by $\sim 22^{\circ}$, the incompatibility between the edges of these two facets is easily bridged by the disordered region connected to the triple line. Comparable adjustments occur at the junction of the $\{111\}$ (brown) facets, although in this case there may also be some residual $\{110\}$ facets present in the vicinity of the triple line. These $\{110\}$ facets are known to roughen and premelt at relatively low temperatures in fcc crystals [23], which may also aid the accommodation of adjacent $\{111\}$ facets across the triple line. It appears, therefore, that the incompatibilities which result from the relative rotation of the two partial polyhedral ECSs that make up the pore are readily accommodated by local atomic rearrangements at the triple line, without any requirement for GB puckering.

There have been several studies which contain photomicrographs of pores at GB fracture surfaces [4-6]. Although the pores shown in these studies are strongly faceted, and therefore have anisotropic surface energies, there is no evidence of significant GB puckering in any of them. Although, one should not generalize the results of the present simulations of pores on a single GB, it is clear from this investigation, as well as from the results of the cited experimental studies, that GB puckering is not necessarily a general phenomenon, and that it should not be expected to appear in every case of GB interactions with pores. 
In contrast to pores at GBs, the case of crystalline particles at GBs can be quite different. The interfaces between crystalline particles and crystalline matrices are more constrained, as they include an accommodation that is generally necessary to account for the mismatch between the two crystalline lattices. Furthermore, the kinetics of accommodation depend on diffusive processes in both the particle and the matrix, which could lead to considerably slower equilibration than in the case of GB pores. Thus it is not surprising that GB puckering has been observed more frequently in the case of particles at GBs [24, 25].

\section{Summary and Conclusions}

Isolated faceted pores and faceted pores at GBs have been studied by MD methods, with the aim of identifying equilibrium configurations, so as to assess the morphological adjustments that occur when pores reside at GBs.

The ECS of isolated faceted pores has been investigated, and found to be subject to the nucleation inhibition of equilibration predicted by Rohrer et al. $[19,20]$ due to the low supersaturation that prevails within non-equilibrated pores. It has been shown that configurations close to the ECS can be obtained if supersaturation is artificially increased by adding mobile adatoms to the internal surfaces of the pores.

The rate of equilibration of pores located at GBs has also been investigated. Although the nucleation energy barriers to facet motion are no longer present for facets in contact with the GB at the triple line, the inhibition of facet motion is still possible for facets that have no contact with the GB. However, this problem can be overcome by approaching the equilibrium shape from different initial configurations.

The configuration of the GB in the vicinity of the pore has been found to be essentially planar, indicating that GB puckering in the vicinity of anisotropic pores is not generally necessary. In the present case, incompatibilities between misoriented facets that meet at the triple line are easily accommodated by local atomic rearrangements of the disordered region of intersection with the GB.

\section{Acknowledgments}

PW wishes to acknowledge the use of resources of the National Energy Research Scientific Computing Center, which is supported by the Office of Science of the U.S. Department of Energy under Contract No. DE-AC02-05CH11231. In addition, the authors wish to thank Dr. David Olmsted for useful discussions, and to acknowledge the Theory and Computer Simulation Department of the CINAM Laboratory in Marseille, for access to their computer facilities. 


\section{$\underline{\text { References }}$}

1. Wulff G. Z Krist Mineral 1901;34:449.

2. Mullins WW. in: Robertson WD, Gjostein NA (Eds.). Metal Surfaces: Structure, Energetics and Kinetics. Cleveland: ASM; 1963.

3. Herring C. in: Gomer R, Smith CS (Eds.). Structure and Properties of Solid Surfaces. Chicago: University of Chicago Press; 1952.

4. Chatain D, Wynblatt P, Rohrer GS. Acta Mater 2005;53:4057.

5. Van Agterveld DTL, Palatzantsas G, De Hosson JTM. Acta Mater 2000;48:1995.

6. Kesternich W, Ullmaier H. J Nucl Mater 2003;312:212.

7. Lee JK, Aaronson HI. Acta Metall 1975;23:799.

8. Lee JK, Aaronson HI. Acta Metall 1975;23:809.

9 Siem EJ, Carter WC, Chatain D. Phil Mag 2004;84:991.

10. Zucker RV, Chatain D, Dahmen U, Hagege S, Carter WC. J Mater Sci 2012;47:8290.

11. Plimpton SJ, J Comp Phys 1995;117:1.

12. http://www.cs.sandia.gov/ sjplimp/lammps.html

13. Plimpton SJ, Hendrickson BA. Parallel molecular dynamics with the embedded atom method, in: Broughton J, Bristowe P, Newsam J (Eds.). Materials Theory and Modelling. Pittsburgh: MRS Proceedings 291; 1993.

14. http://www.cs.sandia.gov/ sjplimp/main.html

15. Foiles SM, Hoyt JJ. Acta Mater 2006;54:3351.

16. Olmsted DL, Foiles SM, Holm EA, Acta Mater 2009;57:3694.

17. Winterbottom WL. Acta Metall 1967;15:303.

18. See for example: Clusters of Atoms and Molecules, Haberland H (Ed.). Berlin: Springer 1994.

19. Mullins WW, Rohrer GS. J Am Ceram Soc 2000;83:214.

20. Rohrer GS, Rohrer CL, Mullins WW. J Am Ceram Soc 2001;84:2099.

21. Herring C. in: Gomer R (Ed.). The Physics of Powder Metallurgy. New York: McGrawHill; 1949.

22. Du D, Zhang H, Srolovitz JD, Acta Mater 2007;55:467.

23. Landa A, Wynblatt P, Hakkinen H, Barnett RN, Landman U. Phys Rev B 1995;51:10972.

24. Dahmen U, Hagège S, Faudot F, Radetic T, Johnson E. Phil Mag 2004;25-26:2651

25. Mizoguchi T, Dahmen U. Phil Mag Let 2009;89:104. 


\section{Figures}

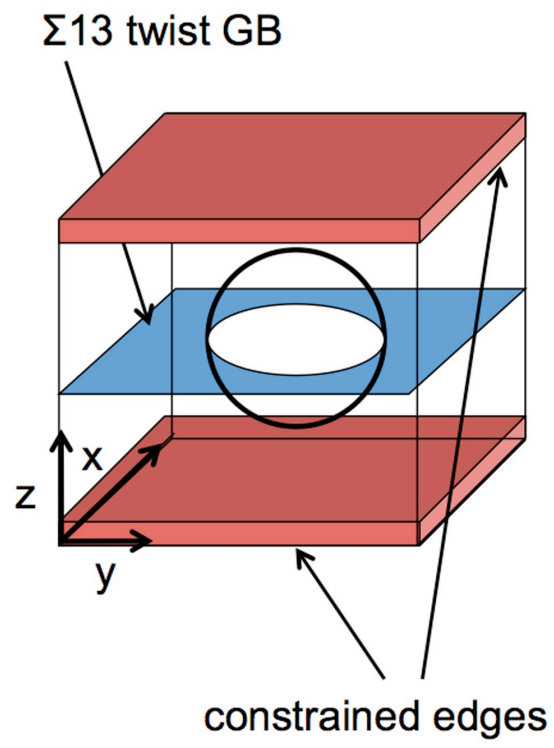

Figure 1. Schematic of computation cell with an initially spherical pore.

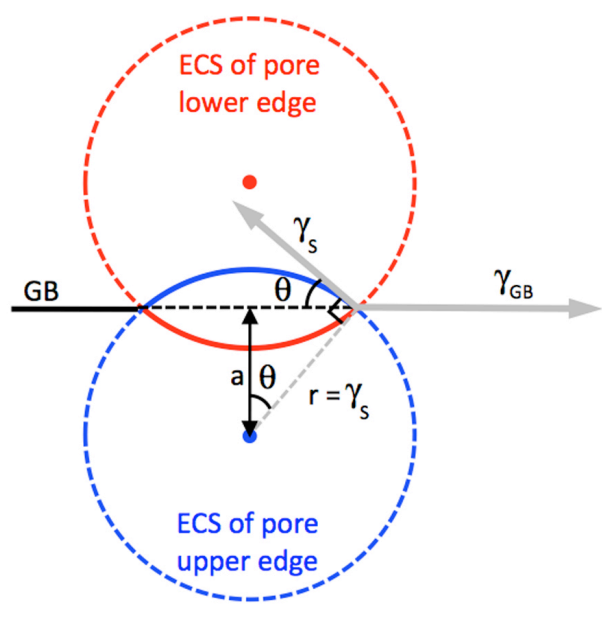

(a)

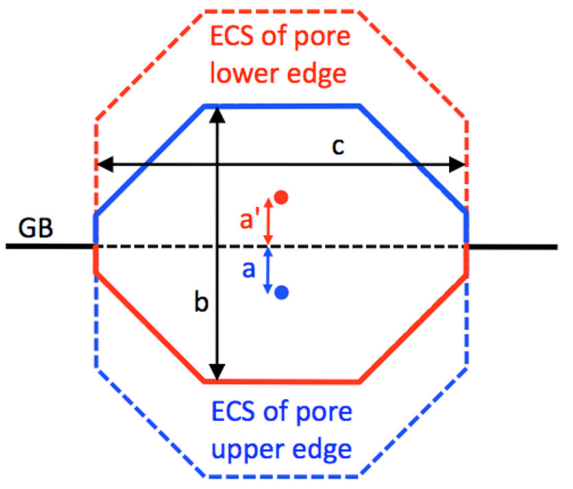

(b)

Figure 2. (a) Schematic of an isotropic pore at a GB: the ECSs of the pore edges are shown by the dashed circles (in red and blue for the pore lower and upper edges, respectively, color on line), $a$ is the distance from the Wulff point to the GB plane, $\theta$ is half the equilibrium dihedral angle of the pore at the GB, and $\gamma_{s}$ and $\gamma_{G B}$ are the interfacial energies of the pore surface and GB respectively. (b) Schematic of an anisotropic pore at a GB: the ECSs of the pore edges are shown by the dashed lines (in red and blue for the lower and upper edges of the pore, respectively), $a^{\prime}$ and $a$ are the distances from the Wulff points of the ECSs of the lower and upper edges of the pore, $b$ is the distance between the upper and lower $\{100\}$ surfaces at the pore edges, and $c$ is the distance between the $\{100\}$ surfaces of the ECS. 


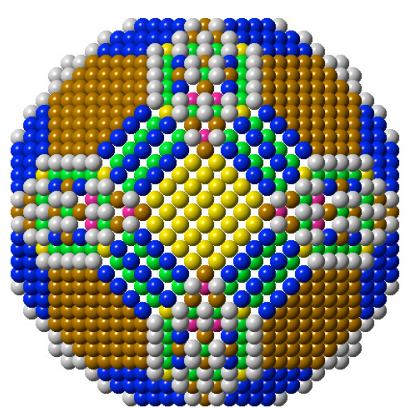

(a)

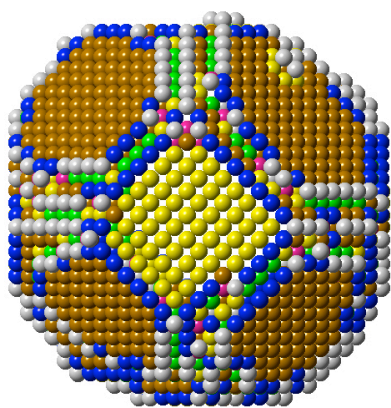

(b)

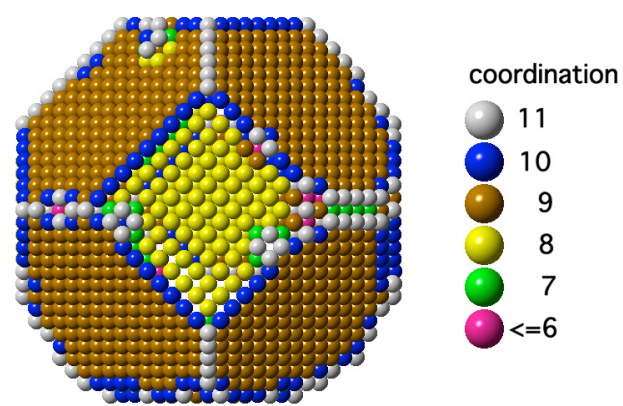

(c)

Figure 3. Pore exterior as viewed along a $<100>$ direction; the color scheme for coordination numbers is shown in the legend (color on-line). (a) Initial configuration of pore before applying $\mathrm{MD}$; (b) pore configuration after equilibration for $20 \mathrm{nsec}$ at $1350 \mathrm{~K}$; (c) configuration after adding 400 atoms to the pore and equilibrating for $12 \mathrm{nsec}$ at $1300 \mathrm{~K}$ (see text).

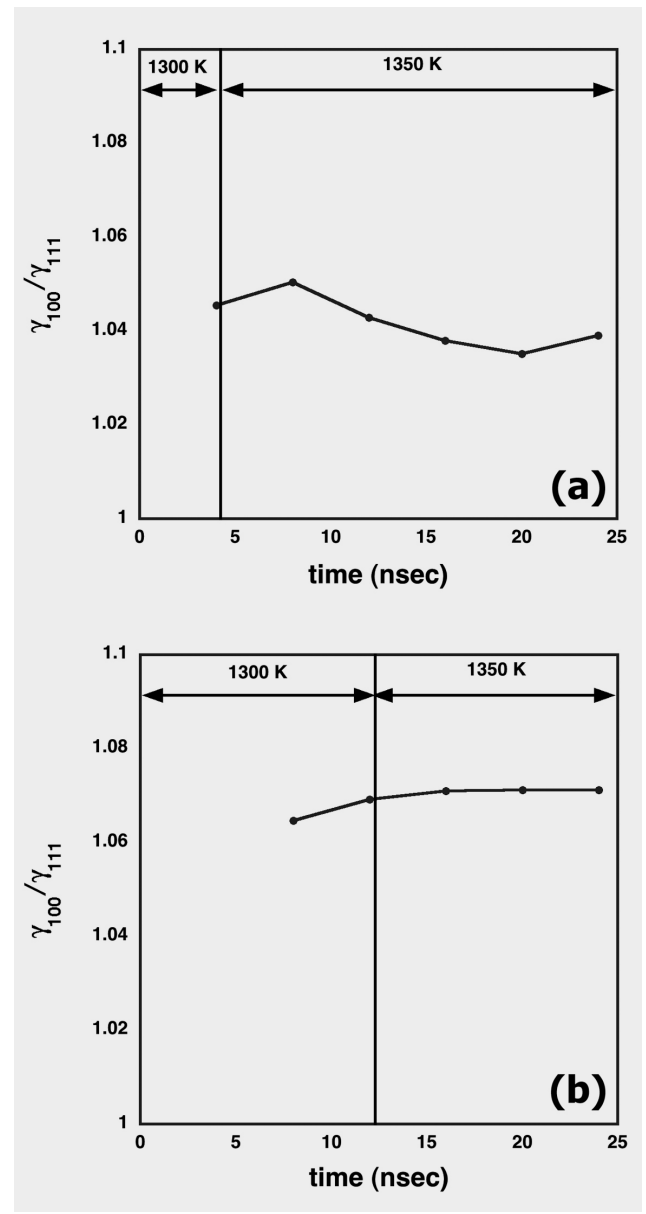

Figure 4. Equilibration of $\gamma_{100} / \gamma_{111}$ as a function of time; (a) for originally spherical pores, and (b) for initially spherical pores after the addition of 400 atoms to the pore surface. Simulations were started at $1300 \mathrm{~K}$, and then continued at $1350 \mathrm{~K}$; the time corresponding to the temperature change is indicated by the vertical lines. 


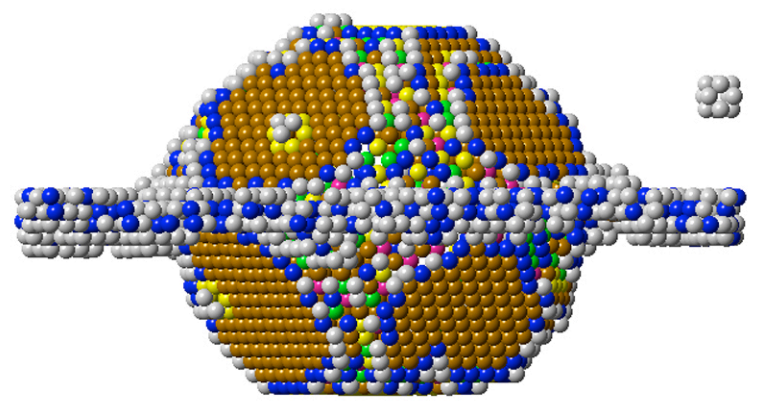

Figure 5. Configuration of an initially spherical pore after equilibration for $28 \mathrm{nsec}$ at $1350 \mathrm{~K}$. The color scheme for identifying atom coordination is the same as in Fig. 3.

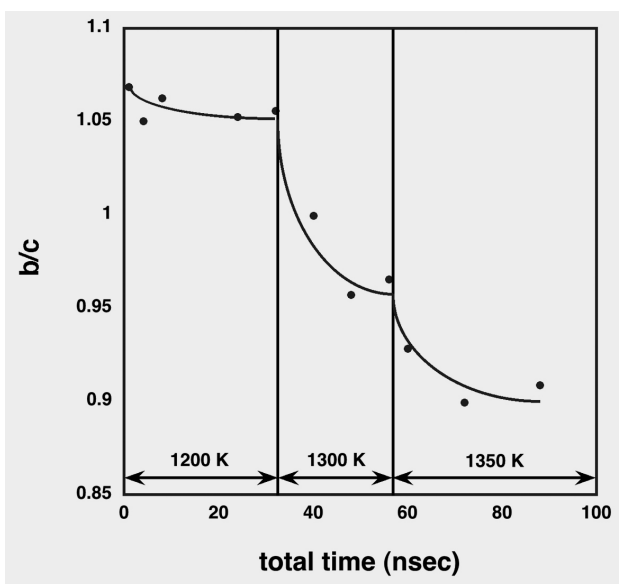

Figure 6. Plot of the ratio $b / c$ vs. total simulation time for the evolution of an initially spherical pore. Simulations were started at $1200 \mathrm{~K}$, and continued at $1300 \mathrm{~K}$ and $1350 \mathrm{~K}$; the time corresponding to temperature changes is indicated by the vertical lines. Curves have been added to aid the eye.

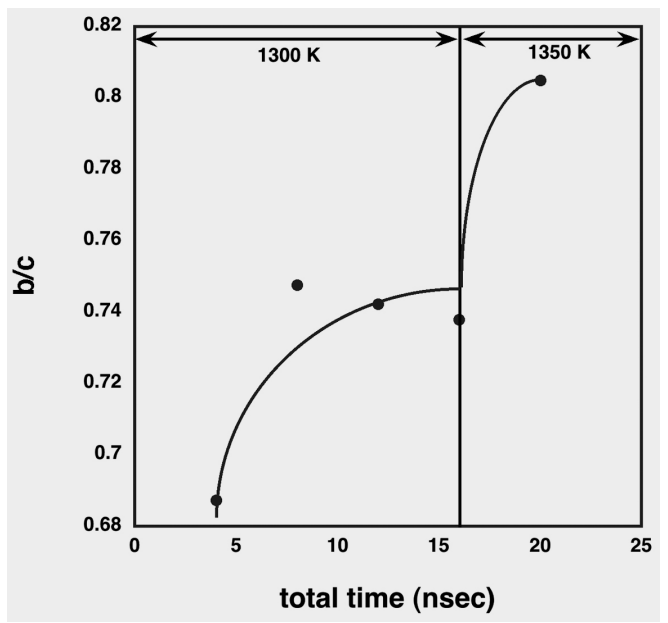

Figure 7. Plot of the ratio $b / c$ vs. total simulation time for the evolution of an initially lenticular pore with $\theta_{\text {eff }}=60^{\circ}$. Simulations were started at $1300 \mathrm{~K}$ and continued at $1350 \mathrm{~K}$ (see text); the time corresponding to temperature changes is indicated by the vertical lines. Curves have been added to aid the eye. 


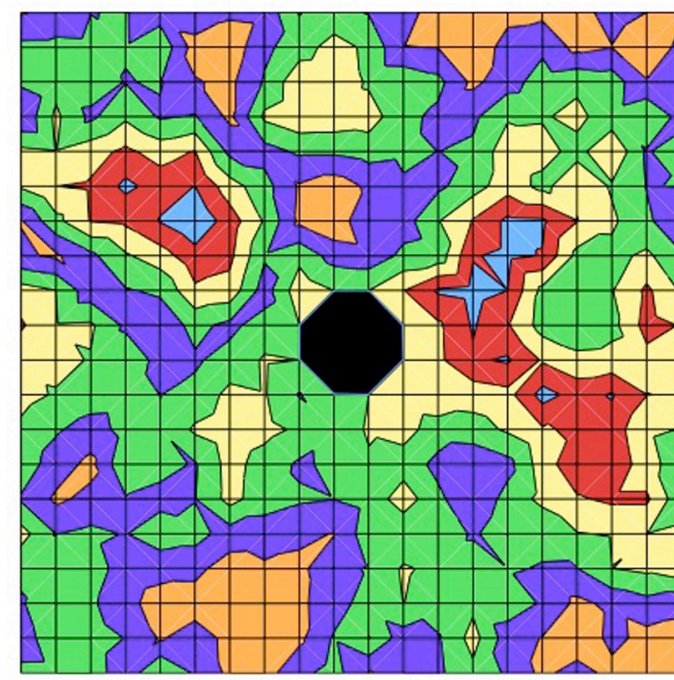

2.0000-3.0000

1.0000-2.0000

$\square 0.0000-1.0000$

$-1.0000-0.0000$

$-2.0000--1.0000$

$-3.0000--2.0000$

Figure 8. Contour plot of GB $\sim 225 \mathrm{~nm}^{2}$ in area surrounding an initially hemispherical void, after equilibration at $1300 \mathrm{~K}$ for $12 \mathrm{nsec}$. The mean height of the boundary is plotted on a 1 $\mathrm{nm} X 1 \mathrm{~nm}$ grid. Heights in $\AA$ are given in the legend. The pore is identified by the central black octagon.

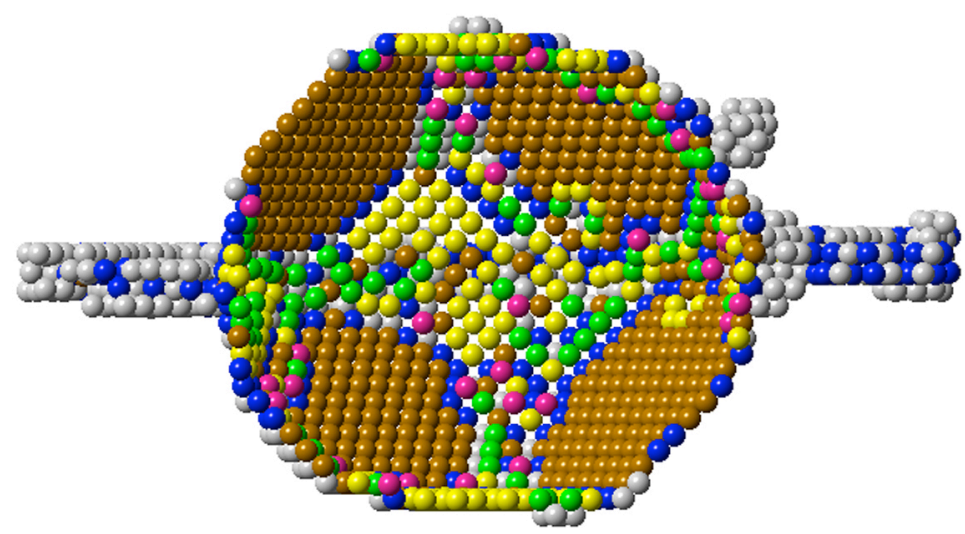

Figure 9. View of pore interior produced by slicing the pore of Fig. 5. The color scheme for identifying atom coordination is the same as in Fig. 3. 